\section{A mutation in separase causes genome instability and increased susceptibility to epithelial cancer}

\author{
Jennifer L. Shepard, 1,3 James F. Amatruda, ,,4 \\ David Finkelstein, ${ }^{1}$ James Ziai, ${ }^{1}$ K. Rose Finley, ${ }^{1}$ \\ Howard M. Stern, ${ }^{1,2}$ Ken Chiang, ${ }^{1}$ \\ Candace Hersey, ${ }^{1}$ Bruce Barut, ${ }^{1}$ \\ Jennifer L. Freeman, ${ }^{2}$ Charles Lee, ${ }^{2}$ \\ Jonathan N. Glickman, ${ }^{2}$ Jeffery L. Kutok, ${ }^{2}$ \\ Jon C. Aster, ${ }^{2}$ and Leonard I. Zon ${ }^{1,5}$ \\ ${ }^{1}$ Children's Hospital, Boston, Massachusetts 02115, USA; \\ ${ }^{2}$ Department of Pathology, Brigham and Women's Hospital, \\ Boston, Massacusetts 02115, USA
}

Proper chromosome segregation is essential for maintenance of genomic integrity and instability resulting from failure of this process may contribute to cancer. Here, we demonstrate that a mutation in the mitotic regulator separase is responsible for the cell cycle defects seen in the zebrafish mutant, cease \& desist (cds). Analysis of $c d s$ homozygous mutant embryos reveals high levels of polyploidy and aneuploidy, spindle defects, and mitotic exit delay. Carcinogenesis studies demonstrated that $c d s$ heterozygous adults have a shift in tumor spectrum with an eightfold increase in the percentage of fish bearing epithelial tumors, indicating that separase is a tumor suppressor gene in vertebrates. These data strongly support a conserved cross-species role for mitotic checkpoint genes in genetic stability and epithelial carcinogenesis.

Supplemental material is available at http://www.genesdev.org.

Received July 18, 2006; revised version accepted November 8, 2006.

\section{Results and Discussion}

A previously described genetic screen in zebrafish for cell proliferation mutants identified cease\&desist (cds), a homozygous lethal mutant with aberrant staining for the phosphorylated form of histone $\mathrm{H} 3(\mathrm{pH} 3)$, a mitotic marker (Shepard et al. 2005). pH3 staining of $c d s$ embryos $36 \mathrm{~h}$ post-fertilization (hpf) shows a slight decrease in the number of $\mathrm{pH} 3$-positive cells (Fig. 1A) and reveals that these pH3-positive foci are also larger than their wild-type sibling counterparts. Further characterization

[Keywords: Cancer; chromosome segregation; mitotic checkpoint; zebrafish]

Present addresses: ${ }^{3}$ Center for Cancer Research, Massachusetts Institute of Technology, Cambridge, MA 02139, USA; ${ }^{4}$ Departments of Pediatrics and Molecular Biology, University of Texas Southwestern Medical Center, 5323 Harry Hines Blvd., Dallas, TX 75390, USA.

${ }^{5}$ Corresponding author.

E-MAIL zon@enders.tch.harvard.edu; FAX (617) 730-0222.

Article is online at http://www.genesdev.org/cgi/doi/10.1101/gad.1470407. of homozygous cds embryos shows that they also exhibit increased apoptosis as early as $24 \mathrm{hpf}$, as detected by TUNEL staining (Fig. 1B). 5-bromo-2-deoxyuridine (BrdU) incorporation also indicates that at $28 \mathrm{hpf}, \mathrm{cds}$ embryos have markedly decreased embryonic proliferation (Supplementary Fig. S1). To further characterize the larger foci phenotype and cell cycle defects exhibited by cds mutant embryos, DNA content analysis was performed on disaggregated 24-hpf embryos. This study demonstrated that $c d s$ embryos have a population of cells with $8 \mathrm{~N}$ DNA content, indicating the presence of polyploid cells (Fig. 1C). Further analysis also demonstrated the presence of $16 \mathrm{~N}$ cells (data not shown). These data suggest that $c d s$ mutants have a fundamental problem with control of the mitotic checkpoint.

To discover the gene responsible for the $c d s$ phenotype, a positional cloning project was undertaken and $c d s$ was mapped and localized to linkage group 6 in a 6.5-centimorgan (cM) interval between the microsatellite markers z5294 and z265 (Fig. 1D). Scanning of additional microsatellite makers narrowed the interval to 0.5 cM. A chromosomal walk was initiated from z11919, and led to the identification of a single BAC clone that contained the $c d s$ locus. Analysis of BAC sequence and human/zebrafish syntenic relationships led to the discovery that the zebrafish ortholog of the vertebrate gene separase was present in this critical interval. Sequence analysis of the cds mutant locus identified a C-to-A transversion introducing a nonsense mutation that is predicted to truncate the zebrafish separase gene after amino acid 597, which would cause loss of the catalytic domain and loss of half of the ARM repeats at the $\mathrm{N}$ terminus (Viadiu et al. 2005). Separase is a cysteine protease that, during mitosis, is responsible for cleaving the cohesin complex that binds sister chromatids together (Nasmyth 2001). Separase is tightly regulated by an anaphase-promoting complex target protein, securin, which has been shown to act as the oncogene pituitary tumor transforming gene (PTTG) in humans (Zou et al. 1999). Separase is also regulated by autocatalytic cleavage and inhibitory phosphorylation (Stemmann et al. 2001; Waizenegger et al. 2002; Zou et al. 2002). Additionally, in yeast, separase has been shown to act as part of the mitotic exit network (MEN) complex, suggesting that separase simultaneously triggers anaphase by cohesin cleavage and, independently of its protease function, initiates mitotic exit through activation of Cdc14 (Stegmeier et al. 2002). Zebrafish separase encodes a 2181-amino-acid protein that is $34 \%$ identical to human separase, and $13 \%$ identical to Schizosaccharomyces pombe Cut1 and Saccharomyces cervisiae Esp1 (Supplementary Fig. S2). The catalytic residues that are present in all caspasehemoglobinase fold family members are also conserved (Aravind and Koonin 2002). DNA content analysis of wild-type embryos injected with antisense morpholinomodified oligonucleotides (morpholinos) targeted to the separase exon 7 splice donor shows that $\sim 30 \%$ of the cells are polyploid, which is similar to the level seen in $c d s$ homozygous mutants (Fig. 1E). These data strongly suggest it is a loss of function of separase that is responsible for the $c d s$ phenotype.

Loss of separase in yeast, human, and mouse cells has 

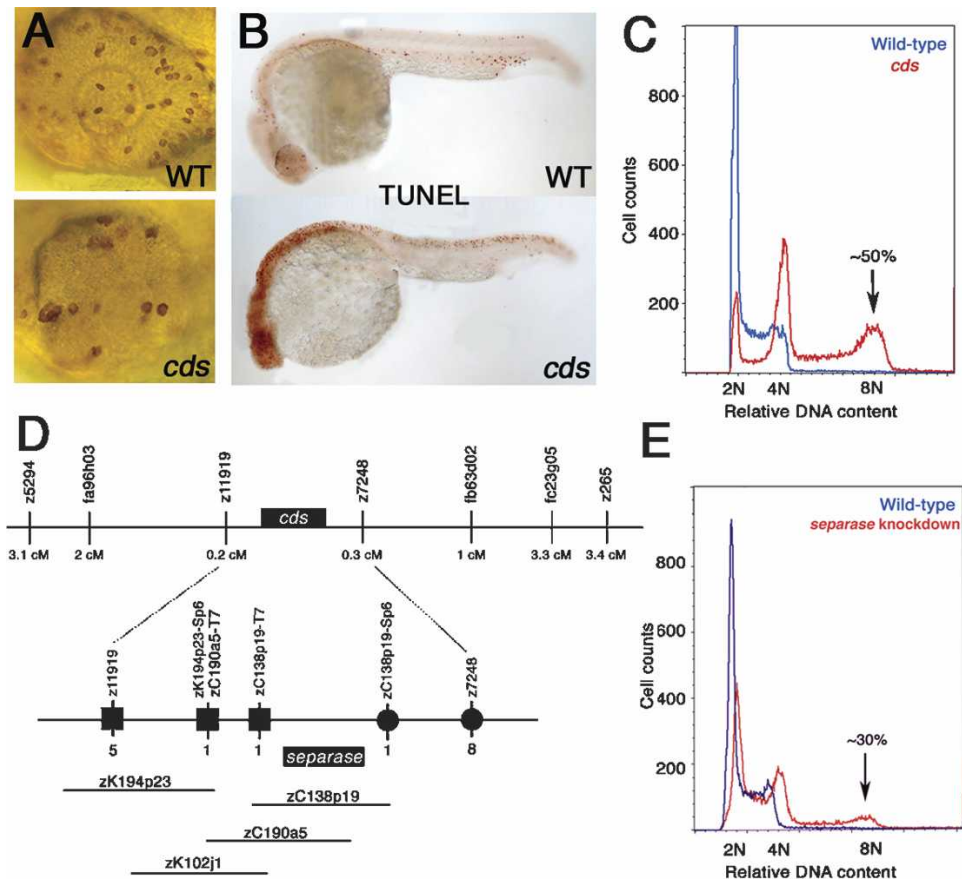

$E$

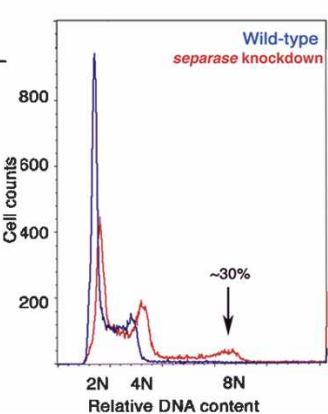

Figure 1. cds has mitotic cells with increased size of $\mathrm{pH} 3$ foci and increased apoptosis and polyploidy, and is caused by a mutation in the zebrafish separase gene. (A) High-power views of a 36-hpf cds mutant show that $\mathrm{pH} 3$-positive foci in $c d s$ are larger in size than in wild-type siblings. (B) TUNEL staining of 24-hpf $c d s$ embryos shows increased apoptosis in the head and dorsal part of the tail. $(C)$ DNA content analysis of $c d s$ homozygous 36-hpf embryos and wild-type siblings showing the presence of polyploid cells in the mutant. $(D)$ Positional cloning of the $c d s$ gene. The $c d s$ locus is depicted by a black bar between the flanking markers z11919 and z7248 identified by analysis of $~ 3000$ meioses from diploid mutants. Below is an enlarged view of the cds locus that depicts the BAC genomic clones identified in a chromosomal walk. The number of recombination events identified from the distal side (squares) and proximal side (circles) are indicated. (E) Phenocopy of the $c d s$ polyploid phenotype by injection of morpholinos targeted to the separase exon 14 splice donor.

been shown to cause mitotic spindle defects such as multiple spindle poles (Baum et al. 1988; Uzawa et al. 1990; Waizenegger et al. 2002; Chestukhin et al. 2003; Kumada et al. 2006; Wirth et al. 2006). Examination of the mitotic spindle in 32-hpf $c d s$ mutants demonstrated that $\sim 50 \%$ of the visible spindles were abnormal (Fig. 2A-I; data not shown). Analysis of $c d s$ cells for $\mathrm{pH} 3$ and mitotic spindles showed that these abnormal mitotic figures do exhibit multipolar spindles some with three to four visible centrosomes (Fig. 2B,D-H). Monopolar spindles are also visible (Fig. 2C). Also present are multipolar spindles that appear to have lobed or distinct nuclei with spindles that may nucleate from shared centrosomes (Fig. 2G,H). These may represent failed mitoses resulting from fused cells or midbody retraction. Another defect commonly seen in cds embryos is lagging chromosomes (Fig. 2I). This phenotype has also been seen in Separase knockdowns in human cells (Chestukhin et al. 2003). Our findings indicate that separase is required for normal chromosome segregation in zebrafish, and supports a conserved role in regulating mitosis in eukaryotic cells.

Studies have shown that the presence of lagging chromosomes slows the spindle elongation rate indicating that cells may detect the presence of such chromosomes and slow anaphase in order to allow the chromosomes extra time to reach the pole (Pidoux et al. 2000). Our studies have demonstrated that such a mitotic exit defect can be detected. To examine the cell cycle timing of $c d s$ cells from $\mathrm{S}$ to $\mathrm{M}$ phase and determine if a mitotic entry or exit defect existed, we performed a BrdU pulse/chase experiment and double stained the embryos with antiBrdU antibody and $\mathrm{pH} 3$ antibody. Examining the timing of when BrdU-positive cells become $\mathrm{pH} 3$ positive allows calculation of the length of time between the $S$ phase and mitotic entry and exit in cds as compared with wild types (Fig. 2J). The S-phase cell population in wild-type embryos begins to enter G2/M $2 \mathrm{~h}$ after BrdU labeling and begins to exit from mitosis at $6 \mathrm{~h}$, with exit completed by $8 \mathrm{~h}$. Cells in $c d s$ embryos enter $M$ phase at the same time as wild types, and also begin to exit at $6 \mathrm{~h}$ post-incorporation. There are still a significant proportion of $c d s$ cells in mitosis at the 8-h time point, indicating slower progression through mitosis, perhaps due to mitotic complications, or a potential mitotic exit defect. Separase has been shown to act as part of a complex that promotes mitotic exit in yeast (Stegmeier et al. 2002), so this phenotype is consistent with a similar role in vertebrates. However, recent studies in mouse embryonic fibroblasts lacking Separase have not uncovered any mitotic exit defects (Kumada et al. 2006). This discrepancy between zebrafish embryos and mouse embryonic fibroblasts (MEFs) may be attributed to studying this phenomenon in a whole organism versus in a cell culture environment; the defect may only be visible in the former.

To further characterize the polyploidy seen in $c d s$ embryos and to look for further evidence of chromosomal instability as indicated by the presence of grossly abnormal mitoses, interphase fluorescent in situ hybridization (FISH) analysis was performed using probes specific to two randomly chosen linkage groups. This analysis shows that cds embryos contain $48 \%$ polyploid and $34 \%$ aneuploid cells versus $2 \%$ and $7 \%$, respectively, in wild types (Fig. $3 \mathrm{~A}, \mathrm{~B})$. Further analysis of metaphase spreads demonstrated the presence of cells in cds mutant embryos with $16 N$ DNA content (Fig. 3C). These cells also contain diplochromosomes (chromosomes that have undergone DNA replication but have not segregated) that appear to remain attached at the centromeres. Diplochromosomes have been previously observed in Drosophila, and more recently in mouse, separase mutants (Jager et al. 2001; Kumada et al. 2006). This confirms the role of vertebrate Separase in cleavage of the bonds between sister chromatids during mitotis.

Given Securin's role as an oncogene (Zou et al. 1999) and that mutation of separase affects chromosome segregation and chromosomal stability, we next asked whether the separase mutation affected cancer susceptibility in heterozygous adults. Twenty-eight-day-old zebrafish (heterozygotes and wild-type siblings) were subjected to a single dose of the carcinogen N-methyl-N' nitro-N-nitrosoguanidine (MNNG). At 3, 6, or 12 mo after exposure, the 663 treated fish were sacrificed, and serial step sections of these fish were examined for the presence of tumors. Each fish was genotyped using a restriction fragment length polymorphism (RFLP) specific 

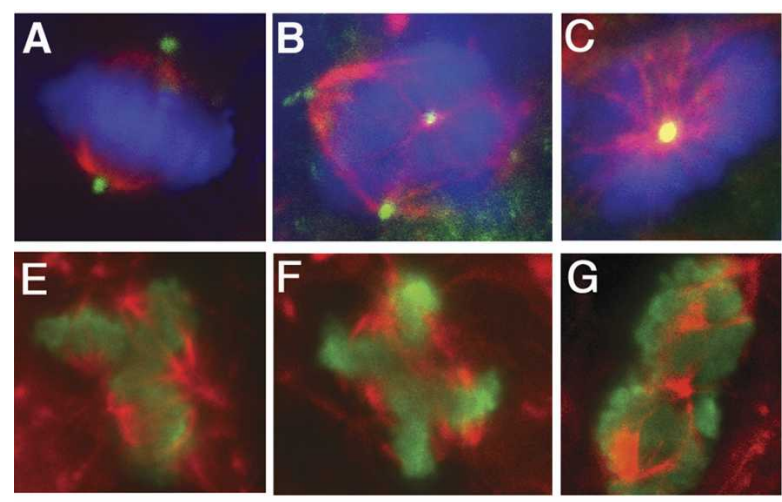
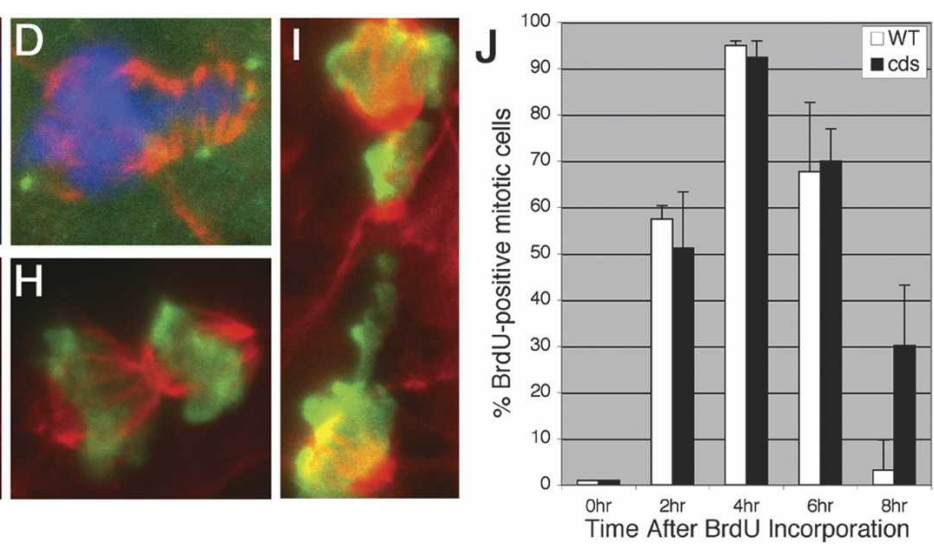

Figure 2. Abnormal mitoses and a mitotic exit defect are present in $c d s .(A-D)$ Mitotic spindles ( $\alpha$-tubulin, red), centrosomes $(\gamma$-tubulin, green), and DNA (DAPI, blue) seen in $c d s$ embryos. (E-I) Mitotic figures (pH3 staining, green) and spindles ( $\alpha$-tubulin, red) seen in $c d s$ embryos. $A$ shows a normal bipolar mitotic spindle. (J) Time course of $c d s$ and wild-type movement from $\mathrm{S}$ phase into/out of G2/M as shown by BrdU incorporation followed by double $\mathrm{pH} 3 / \mathrm{BrdU}$ staining at varying time points post-incorporation.

to the separase mutant allele. cds heterozygotes have a 2.5 -fold increase in cancer susceptibility relative to wildtype siblings after MNNG exposure (Fig. 4A; $p=0.005$ ). These studies demonstrate that separase mutations increased cancer susceptibility in zebrafish, and this is the first demonstration of separase as a tumor suppressor gene.

Work in other organisms has shown that certain genetic mutations may cause an increased incidence of a particular tumor type. To address this issue in the $c d s$ heterozygotes, analysis of the tumor spectrum in $c d s$ heterozygotes and wild types after MNNG exposure was performed. This investigation shows that treated $c d s$ heterozygotes have an approximately eightfold increase in the percentage of $c d s$ heterozygous fish with epithelial tumors as compared with treated wild types (Fig. 4B, $p=0.001)$. The level of epithelial tumors seen in $c d s$ heterozygotes is also higher than that seen in MNNGtreated fish in previous studies (Spitsbergen et al. 2000; Shepard et al. 2005). These epithelial tumors consist of, in part, intestinal adenomas (Fig. 4C,D) and cholangiocarcinomas (Fig. 4E,F). Epithelial neoplasms, including adenomas and carcinomas, are common tumors in human adults, and the rates of such cancers have been shown to increase with age (Chang et al. 2001; American Cancer Society 2004). Another feature of epithelial tumors in humans is their aberrant cytogenetic profiles with visible aneuploidy and chromosomal amplification, deletions, and translocations (Atkin 1986, 1989). Genomic instability caused by the heterozygous loss of separase in zebrafish may be the causative factor in the shift in the tumor spectrum toward epithelial neoplasms. Interestingly, loss of separase in Drosophila has been shown to cause defects in epithelial organization and integrity (Pandey et al. 2005). A specific role for separase in epithelial cells may also provide an explanation for this shift in tumor spectrum. Consistent with the findings presented here, Sotillo et al. (2006) have shown recently that Mad2 overexpression, which leads to Securin stabilization and Separase inhibition, results in spontaneous tumor initiation in mice with a tumor spectrum similar to that seen in separase heterozygous zebrafish.

Heterozygous mutations in tumor suppressor genes often contribute to tumor formation through a mechanism of loss of heterozygosity. To determine if this mechanism was applicable to the epithelial tumors arising in the separase heterozygotes, we performed FISH on 10 epithelial tumors from $c d s$ heterozygotes using a probe that contained the separase locus and a centromeric control probe from the same linkage group. In two large and advanced epithelial tumors we found a single copy of separase and two copies of the control centromeric probe (in $\sim 70 \%$ of the cells in each tumor, $n=200$ cells) (Fig. 4G; Supplementary Fig. S4), indicating loss of one allele of the separase gene. Sequence analysis of laser-capture microdissected tissue from the tumor depicted in Figure 4G indicated that the wild-type allele was lost (Supplementary Fig. S5). Areas outside the tumor margins had two copies of each probe (Fig. 4H; Supplementary Fig. S4). It is also possible that loss of function of separase occurs in some of the tumors by a mechanism other than deletion, such as point mutation or silencing, although sequencing of the conserved protease domain in three epithelial tumors did not reveal any mutations (data not shown). There is no apparent polyploidy in the tumor cells with separase loss of heterozygosity (LOH) (Fig. 4G; Supplementary Fig. S4; data not shown). The zebrafish system currently does not have a platform technology to comprehensively assess global chromosomal instability. Until a comprehensive approach is possible, we cannot be certain whether instability exists in these tumors. Our data clearly demonstrates that heterozygous loss of separase contributes to initiation and progression of epithelial tumors. While haploinsufficiency of the separase gene appears to be the mechanism for the increased tumor incidence in the majority of cases, loss of heterozygosity of the separase gene appears to play a role in tumor progression in some tumors. Tumor formation may be achieved by similar mechanisms in both instances. In many environments, complete loss of separase function would likely have a negative effect on cell viability because of gross chromosomal missegregation. It is possible that only after additional mutations have occurred in the tumor that affect the cell cycle and mitotic control would loss of the functional allele of separase give a selective advantage to the tumor cells and advance tumor progression. Therefore, in more advanced tumors, complete loss of function could provide a competitive advantage for the tumor cells, enough to see $\mathrm{LOH}$ at some frequency. The absence of polyploidy in these tumors suggests that the cells have developed a 

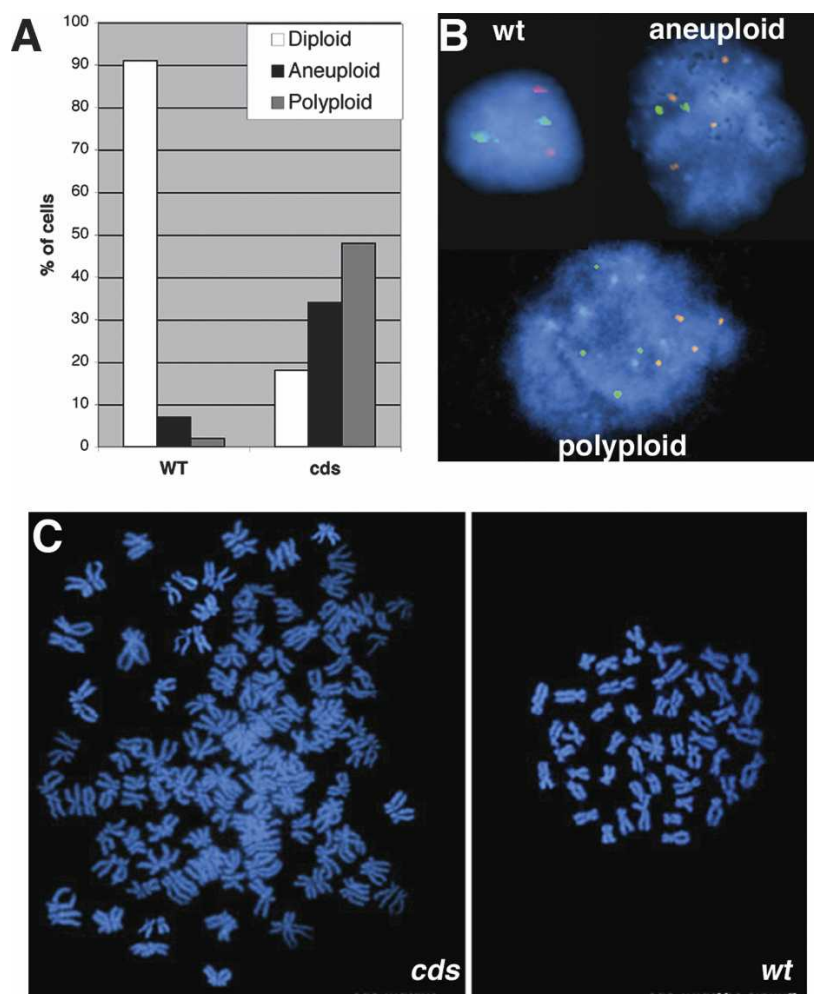

Figure 3. cds mutant embryos demonstrate polyploidy and aneuploidy. (A) Percentage of polyploid and aneuploid cells in interphase nuclei of $c d s$ (black bars) and wild-type embryos (white bars). (B Chromosome copy number enumeration for linkage group 2 (red) and linkage group 16 (green). The aneuploid cell shown in $B$ exhibits tetrasomy for linkage group 2 and disomy for linkage group $16 .(C)$ Metaphase spreads from $c d s$ and wild-type embryos.

way to partially compensate for complete loss of separase. This supports our notion that genetic lesions in the advanced tumor leads to a selective advantage for complete separase loss. While LOH is observed in some tumors, our data demonstrates that loss of only one copy of separase is sufficient in most cases to promote tumorigenesis and shift tumor spectrum toward epithelial neoplasms.

These data represent the first link between separase and increased cancer susceptibility and the first evidence that separase acts as a tumor suppressor. The presence of genomic instability and severe mitotic abnormalities in cds homozygous mutant embryos also demonstrates that zebrafish separase functions in a similar capacity to its mammalian and yeast counterparts. It is possible that separase may also act as a tumor suppressor in humans, although initial sequencing of the separase locus in 82 human tumor cells lines representing a myriad of tumor types did not reveal any obvious mutations (data not shown). The identification of Securin as a human oncogene does support a role for this pathway in human tumor formation. A more focused sequencing approach concentrating on tumors of epithelial origin may have to be undertaken in order to reveal such a role in humans.

This work also suggests interesting implications for the study of the interactions between carcinogens and genetic mutations. In zebrafish, carrying separase mutations causes a clear susceptibility to cancers outside the normal tumor spectrum when exposed to the carcinogen, MNNG. Examples of this type of specific genetic susceptibility to carcinogens have already been shown to exist in certain cases (Bennett et al. 1999; Tan et al. 2000). Therefore, in addition to providing the first evidence of separase tumor suppressor function, our studies demonstrate that zebrafish can serve as an excellent model system for the study of epithelial cancers, and could provide insight into the interactions between carcinogens and genetic predispositions.

\section{Material and methods}

Whole-mount immunohistochemistry

pH3 staining, TUNEL staining, BrdU incoporation, DNA content analysis, and mitotic spindle/centromere staining were done as previously described (Shepard et al. 2005).

\section{Genetic mapping, genotyping, and library screens}

$c d s$ wik strains were outcrossed to $A B$ to create polymorphic mapping strains. Low-resolution mapping was done using pools of 40 diploid mutant and 40 diploid wild-type embryos and scanning for linked polymor-
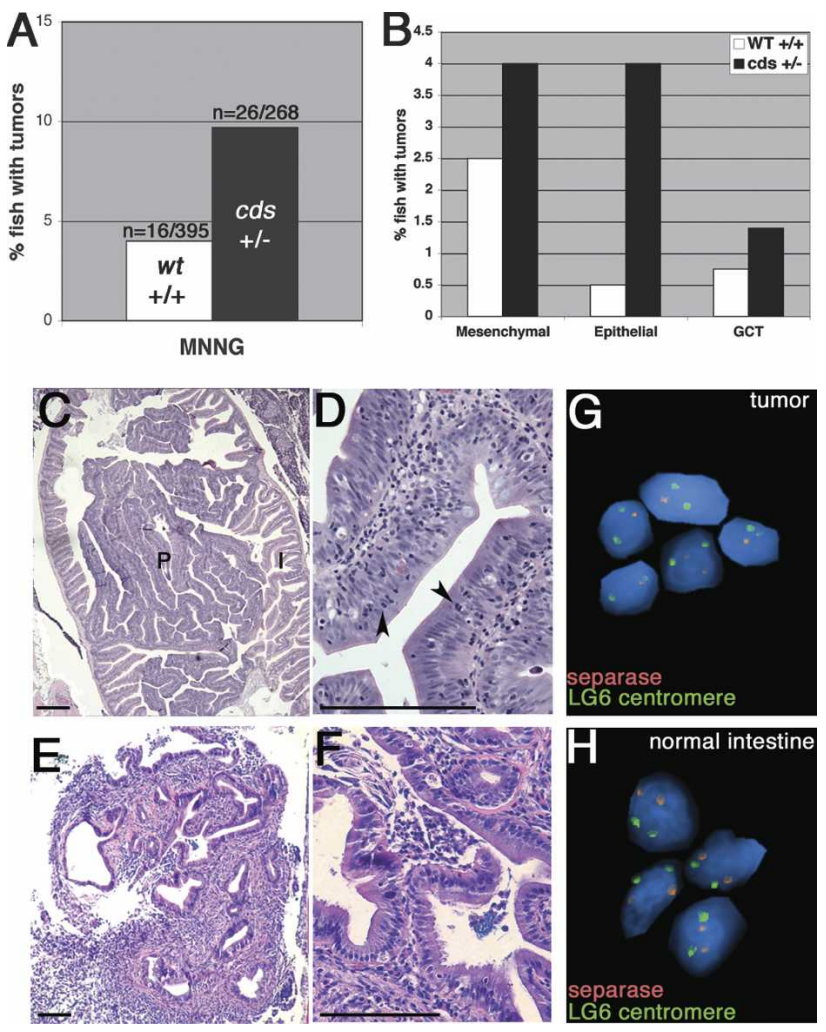

Figure 4. $c d s$ adult heterozygotes have a higher susceptibility to cancer and a distinct tumor spectrum as compared with wild types. (A) After treatment with MNNG, tumors were detected in $9.7 \%$ of $c d s$ heterozygotes and $4 \%$ of sibling wild types. A two-sided Fisher's Exact test gives this finding a $p$ value of 0.005 . (B) Percentage of MNNG-treated $c d s$ and wild-type fish bearing either epithelial, mesenchymal, or germ cell tumors. $(C, D)$ Intestinal adenoma $(P)$ arising as a polyp in otherwise normal intestine (I). Mitotic figures are abundant (arrowheads), and there is nuclear crowding with stratification. $(E, F)$ Ductal adenocarcinoma arising from intestine or pancreas. Bars: $C, F, 100 \mu \mathrm{m} ; D, G, 50 \mu \mathrm{m}$. $(G, H)$ Fluorescent in situ hybridization on paraffin sections seen in $F$ and $G$ using a BAC probe containing the separase gene (orange) and a control LG6 centromeric probe (green). DAPI is in blue. $G$ shows an area within the tumor. $H$ shows an area in the normal intestine. 
phic markers using a range of CA microsatellite markers on each linkage group. After linkage was established to LG 6, CA microsatellites on this linkage group were tested for polymorphism in mapping families and then tested on a medium resolution panel of 88 mutants and eight wild types. Close flanking markers z5294 and z265 were identified, and then the entire mapping panel consisting of $\sim 1500$ diploid $c d s$ embryos was tested with both markers. Recombinants were identified for both markers. ESTs in that region on the RH panel map and additional microsatellite markers were scanned for polymorphisms and close flanking markers of z11919 and z7248 were identified. A chromosomal walk was initiated from z11919. Overgo oligos were designed from BAC end sequence and hybridized to CHORI and DanioKey BAC library filters. Polymorphic markers were identified on BAC ends by SSCP analysis. BAC end sequence was used to identify a BAC in the critical interval that contained the $c d s$ locus and the entire separase gene.

\section{cds RFLP genotyping}

Genomic DNA was extracted from embryos or adult tailclips and amplified with nested PCR primers flanking the $c d s$ mutation (forward primer1, 5'-GACACACGCCTGAGGTAAC-3'; reverse primer1, 5'-CAT AACCCTTAAACATTTTCCTG-3'; forward primer2, 5'-CGGGTTAG GAGAATCCTTAG- ${ }^{\prime}$; reverse primer2, 5'-TGTATGCAAGTCTCGA GTGG-3'). The initial PCR conditions were as follows: $20 \mathrm{sec}$ at $94^{\circ} \mathrm{C}, 30$ sec at $65^{\circ} \mathrm{C}$ (decrease $0.5^{\circ} \mathrm{C}$ per cycle), $1 \mathrm{~min}$ at $72^{\circ} \mathrm{C}$ for 30 cycles and then an additional 10 cycles of $20 \mathrm{sec}$ at $94^{\circ} \mathrm{C}, 30 \mathrm{sec}$ at $58^{\circ} \mathrm{C}$, and $1 \mathrm{~min}$ at $72^{\circ} \mathrm{C}$. The second nested PCR conditions were $20 \mathrm{sec}$ at $94^{\circ} \mathrm{C}, 30 \mathrm{sec}$ at $58^{\circ} \mathrm{C}$, and $1 \mathrm{~min}$ at $72^{\circ} \mathrm{C}$ for 30 cycles. The PCR products were digested for $2.5 \mathrm{~h}$ at $37^{\circ} \mathrm{C}$ with the enzyme Hpy $188 \mathrm{I}$ (New England Biolabs), which cuts the mutant but not the wild-type allele. Digests were run on a $2.5 \%$ agarose gel and the presence of digestion fragments indicated the mutant allele.

\section{Embryo injection experiments}

Morpholinos were designed to the exon 14 splice donor site (5'-ACTG GCTGAGTGTCTGCAGTTTGGT-3') (GeneTools). One-cell stage embryos were injected into the yolk sac with $1 \mathrm{~nL}$ of a $500-\mu \mathrm{M}$ solution of the splice morpholino (or control vehicle). Embryos were examined at 24 hpf for the characteristic morphology of $c d s$ as well as undergoing DNA content analysis.

\section{Cytogenetics}

Metaphase chromosome spreads and interphase nuclei were obtained from 24-hpf embryos using standard cytogenetic procedures. Cells were arrested at metaphase with colchicine, subjected to hypotonic treatments, and fixed onto glass slides with 3:1 methanol:acetic acid. Fluorescence in situ hybridization was performed using standard protocols. Fluorescently labeled probes included a rhodamine-labeled zC039P05 BAC DNA probe for linkage group 2 and a FITC-labeled zC132M17 BAC DNA probe for linkage group 16. For tumor sections, the separase locus probe was $\mathrm{zC} 233 \mathrm{~B} 16$ and the centromere probe was $\mathrm{zK} 11 \mathrm{~J} 4$.

\section{Carcinogenesis}

Twenty-eight-day-old fry from backcrosses of mutant heterozygotes were exposed to MNNG (Spitsbergen et al. 2000) and analyzed as previously described (Shepard et al. 2005).

\section{Statistical methods}

A Fisher exact test was used to assess whether tumor susceptibility differed between $c d s$ heterozygotes and wild-type zebrafish. All time points were combined for this calculation. All $p$ values are two sided.

\section{References}

American Cancer Society. 2004. Cancer facts and figures 2004. American Cancer Society, Inc., Atlanta, GA.

Aravind, L. and Koonin, E.V. 2002. Classification of the caspase-hemoglobinase fold: Detection of new families and implications for the origin of the eukaryotic separins. Proteins 46: 355-367.

Atkin, N.B. 1986. Lack of reciprocal translocations in carcinomas. Cancer Genet. Cytogenet. 21: 275-278.

Atkin, N.B. 1989. Solid tumor cytogenetics. Progress since 1979. Cancer Genet. Cytogenet. 40: 3-12.

Baum, P., Yip, C., Goetsch, L., and Byers, B. 1988. A yeast gene essential for regulation of spindle pole duplication. Mol. Cell. Biol. 8: 5386-5397.
Bennett, W.P., Alavanja, M.C., Blomeke, B., Vahakangas, K.H., Castren, K., Welsh, J.A., Bowman, E.D., Khan, M.A., Flieder, D.B., and Harris, C.C. 1999. Environmental tobacco smoke, genetic susceptibility, and risk of lung cancer in never-smoking women. J. Natl. Cancer Inst. 91: 2009-2014.

Chang, S., Khoo, C., and DePinho, R.A. 2001. Modeling chromosomal instability and epithelial carcinogenesis in the telomerase-deficient mouse. Semin. Cancer Biol. 11: 227-239.

Chestukhin, A., Pfeffer, C., Milligan, S., DeCaprio, J.A., and Pellman, D. 2003. Processing, localization, and requirement of human separase for normal anaphase progression. Proc. Natl. Acad. Sci. 100: 45744579.

Jager, H., Herzig, A., Lehner, C.F., and Heidmann, S. 2001. Drosophila separase is required for sister chromatid separation and binds to PIM and THR. Genes \& Dev. 15: 2572-2584.

Kumada, K., Yao, R., Kawaguchi, T., Karasawa, M., Hoshikawa, Y., Ichikawa, K., Sugitani, Y., Imoto, I., Inazawa, J., Sugawara, M., et al. 2006. The selective continued linkage of centromeres from mitosis to interphase in the absence of mammalian separase. J. Cell Biol. 172: 835-846.

Nasmyth, K. 2001. Disseminating the genome: Joining, resolving, and separating sister chromatids during mitosis and meiosis. Annu. Rev. Genet. 35: 673-745.

Pandey, R., Heidmann, S., and Lehner, C.F. 2005. Epithelial re-organization and dynamics of progression through mitosis in Drosophila separase complex mutants. J. Cell Sci. 118: 733-742.

Pidoux, A.L., Uzawa, S., Perry, P.E., Cande, W.Z., and Allshire, R.C. 2000. Live analysis of lagging chromosomes during anaphase and their effect on spindle elongation rate in fission yeast. J. Cell Sci. 113: 4177-4191.

Shepard, J.L., Amatruda, J.F., Stern, H.M., Subramanian, A., Finkelstein, D., Ziai, J., Finley, K.R., Pfaff, K.L., Hersey, C., Zhou, Y., et al. 2005. A zebrafish bmyb mutation causes genome instability and increased cancer susceptibility. Proc. Natl. Acad. Sci. 102: 13194-13199.

Sotillo, R., Hernando, E., Diaz-Rodriguez, E., Teruya-Feldstein, J., Cordon-Cardo, C., Lowe, S.W., and Benezera, R. 2006. Mad2 overexpression promotes aneuploidy and tumorigenesis in mice. Cancer Cell (in press).

Spitsbergen, J.M., Tsai, H.W., Reddy, A., Miller, T., Arbogast, D., Hendricks, J.D., and Bailey, G.S. 2000. Neoplasia in zebrafish (Danio rerio) treated with $\mathrm{N}$-methyl-N'-nitro-N-nitrosoguanidine by three exposure routes at different developmental stages. Toxicol. Pathol. 28: 716-725.

Stegmeier, F., Visintin, R., and Amon, A. 2002. Separase, polo kinase, the kinetochore protein Slk19, and Spo12 function in a network that controls Cdc14 localization during early anaphase. Cell 108: 207-220.

Stemmann, O., Zou, H., Gerber, S.A., Gygi, S.P., and Kirschner, M.W. 2001. Dual inhibition of sister chromatid separation at metaphase. Cell 107: 715-726.

Tan, W., Song, N., Wang, G.Q., Liu, Q., Tang, H.J., Kadlubar, F.F., and Lin, D.X. 2000. Impact of genetic polymorphisms in cytochrome P450 2E1 and glutathione S-transferases M1, T1, and P1 on susceptibility to esophageal cancer among high-risk individuals in China. Cancer Epidemiol. Biomarkers Prev. 9: 551-556.

Uzawa, S., Samejima, I., Hirano, T., Tanaka, K., and Yanagida, M. 1990. The fission yeast cut $1+$ gene regulates spindle pole body duplication and has homology to the budding yeast ESP1 gene. Cell 62: 913-925.

Viadiu, H., Stemmann, O., Kirschner, M.W., and Walz, T. 2005. Domain structure of separase and its binding to securin as determined by EM. Nat. Struct. Mol. Biol. 12: 552-553.

Waizenegger, I., Gimenez-Abian, J.F., Wernic, D., and Peters, J.M. 2002. Regulation of human separase by securin binding and autocleavage. Curr. Biol. 12: 1368-1378.

Wirth, K.G., Wutz, G., Kudo, N.R., Desdouets, C., Zetterberg, A., Taghybeeglu, S., Seznec, J., Ducos, G.M., Ricci, R., Firnberg, N., et al. 2006. Separase: A universal trigger for sister chromatid disjunction but not chromosome cycle progression. J. Cell Biol. 172: 847-860.

Zou, H., McGarry, T.J., Bernal, T., and Kirschner, M.W. 1999. Identification of a vertebrate sister-chromatid separation inhibitor involved in transformation and tumorigenesis. Science 285: 418-422.

Zou, H., Stemman, O., Anderson, J.S., Mann, M., and Kirschner, M.W. 2002. Anaphase specific auto-cleavage of separase. FEBS Lett. 528: 246-250. 


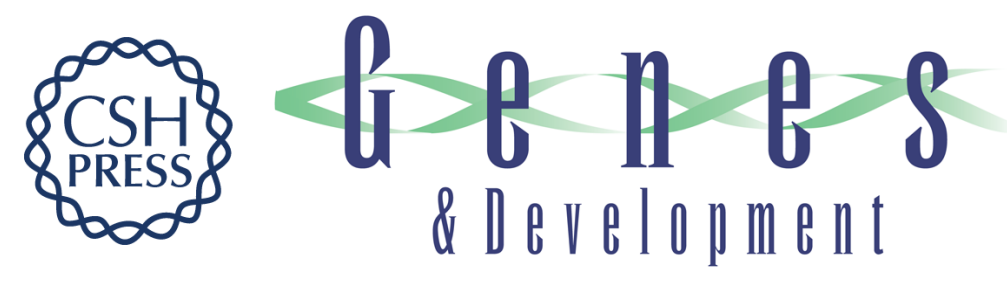

\section{A mutation in separase causes genome instability and increased susceptibility to epithelial cancer}

Jennifer L. Shepard, James F. Amatruda, David Finkelstein, et al.

Genes Dev. 2007, 21:

Access the most recent version at doi:10.1101/gad.1470407

Supplemental
Material http://genesdev.cshlp.org/content/suppl/2007/01/18/21.1.55.DC1

References This article cites 23 articles, 10 of which can be accessed free at:

http://genesdev.cshlp.org/content/21/1/55.full.html\#ref-list-1

License

Email Alerting Receive free email alerts when new articles cite this article - sign up in the box at the top

Service right corner of the article or click here.

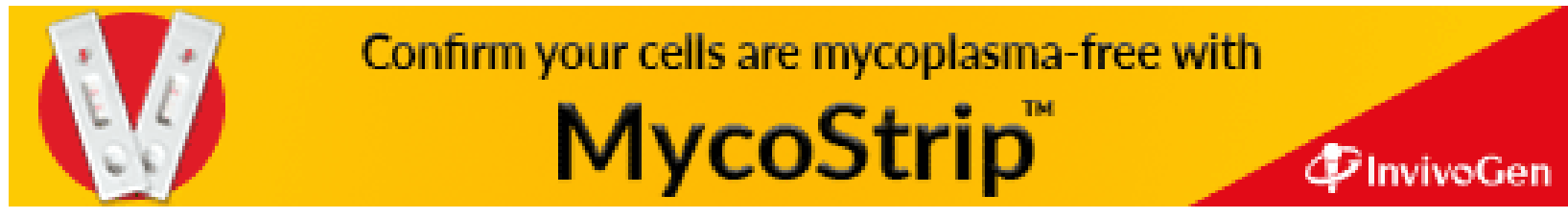

